\title{
Tratamento artroscópico do impacto femoroacetabular na epifisiólise capital femoral proximal: Relato de caso*
}

\section{Arthroscopic Treatment of Femoroacetabular Impingement in Slipped Capital Femoral Epiphysiolysis: A Case Report}

\author{
Marco Antonio Pedroni ${ }^{10}$ Ademir Antonio Schuroff ${ }^{10}$ Rafael Wei Min Leal Chang ${ }^{1,2(0)}$ \\ Bruno Bellaguarda Batista ${ }^{2}$ \\ ${ }^{1}$ Serviço de Cirurgia do Quadril, Departamento de Ortopedia e \\ Traumatologia, Hospital Universitário Cajuru (HUC), Pontifícia \\ Universidade Católica do Paraná (PUCPR), Curitiba, PR, Brasil \\ 2 Serviço de Ortopedia e Traumatologia, Hospital Universitário \\ Getúlio Vargas (HUGV), Universidade Federal do Amazonas (UFAM), \\ Manaus, AM, Brasil

\begin{abstract}
Endereço para correspondência Rafael Wei Min Leal Chang, Serviço de Ortopedia e Traumatologia, Hospital Universitário Getúlio Vargas (HUGV), Universidade Federal do Amazonas (UFAM), Rua Tomas de Vila Nova, 4, Nossa Sra. das Graças, Manaus, AM, 69020-170, Brasil
\end{abstract} \\ (e-mail: rafchang@gmail.com).
}

Rev Bras Ortop 2021;56(1):121-124.

\section{Resumo \\ Palavras-chave \\ - artroscopia \\ - quadril \\ - epífise deslocada \\ - impacto femoroacetabular}

A epifisiólise capital femoral proximal (ECFP) pode resultar em impacto femoroacetabular (IFA) do quadril em até um terço dos casos. A deformidade residual em came ou "cabo de pistola" está associada a lesão condrolabral, resultando em dor, incapacidade funcional, e osteoartrose precoce. O tratamento artroscópico com osteocondroplastia mostrou-se benéfico em um caso selecionado de IFA secundário a ECFP.

Slipped capital femoral epiphysiolysis (SCFE) may result in femoroacetabular impingement (FAI) of the hip in up to one third of the cases. Residual deformity of the cam-type, or "pistol-grip", is associated with chondrolabral injury, resulting in pain, functional disability, and early osteoarthritis. The arthroscopic treatment with osteochondroplasty proved to be beneficial in a selected case of FAI secondary to SCFE.
Trabalho desenvolvido no Departamento de Ortopedia e Traumatologia, Pontifícia Universidade Católica do Paraná (PUCPR), Curitiba, PR, Brasil.

recebido 29 de Novembro de 2019 aceito

15 de Abril de 2020
DOI https://doi.org/ $10.1055 / \mathrm{s}-0040-1714222$ ISSN 0102-3616.

\section{Introdução}

A epifisiólise capital femoral proximal (ECFP) é o transtorno mais comum do quadril do adolescente, com uma incidência reportada de 10.8 em cada 100 mil habitantes. A (c) 2021. Sociedade Brasileira de Ortopedia e Traumatologia. All rights reserved.

This is an open access article published by Thieme under the terms of the Creative Commons Attribution-NonDerivative-NonCommercial-License, permitting copying and reproduction so long as the original work is given appropriate credit. Contents may not be used for commercial purposes, or adapted, remixed, transformed or built upon. (https://creativecommons.org/ licenses/by-nc-nd/4.0/)

Thieme Revinter Publicações Ltda., Rua do Matoso 170, Rio de Janeiro, RJ, CEP 20270-135, Brazil 
bilateralidade pode ocorrer em até $20 \%$ dos casos. ${ }^{1}$ Fatores mecânicos como obesidade, retroversão femoral e relativa orientação vertical da fise proximal do fêmur foram associados à etiologia. ${ }^{2}$

O colo femoral proximal desloca-se anterolateralmente, no nível da fise, sobre a cabeça femoral, que permanece dentro do acetábulo. Essa deformidade leva a uma proeminência no aspecto anterolateral da transição cefalocervical e a uma atitude em rotação externa do fêmur proximal. Os pacientes subsequentemente podem desenvolver uma deformidade em "cabo de pistola" próxima à cabeça do fêmur, também denominada "queilo" por alguns autores. ${ }^{3}$ Essa deformidade pode sofrer melhora por remodelamento, mas tal potencial é limitado pela fixação in situ, que compromete o crescimento fisário. Além disso, a ECFP ocorre numa faixa etária em que a capacidade de compensação de deformidades residuais por remodelamento não é mais possível.

Até um terço dos pacientes com diagnóstico de ECFP apresentam dor persistente e/ou impacto femoroacetabular (IFA) resultante da deformidade. ${ }^{4}$ A proeminência residual (deformidade em "cabo de pistola") e a relativa retroversão da cabeça femoral foram definidas como causa de IFA tipo came, com piores resultados clínicos e radiográficos em longo prazo. Um marco importante dessa deformidade consiste na compensação reduzida ou ausente entre a cabeça femoral e o colo, que pode ser graduada radiograficamente.

A proeminência residual na junção cabeça-colo se projeta no rebordo acetabular, gerando estresse na junção condrolabral, resultando na separação do lábrum da cartilagem articular, a qual é precursora à lesão condral irreversível. Essa lesão se inicia logo após o deslizamento na ECFP, e costuma progredir com o tempo, levando à deterioração do quadril em idades precoces. $^{5}$

Há evidências na literatura que apoiam a osteocondroplastia artroscópica do colo femoral no tratamento do IFA sintomático secundário a ECFP, com resultados animadores,, 7 e é sugerida a abordagem precoce logo após o deslizamento, a fim de se prevenir contra a progressão irreversível com piores resultados em longo prazo. ${ }^{7}$

\section{Relato do Caso}

Paciente do sexo feminino, de 15 anos de idade, sem comorbidades, no $2^{\circ}$ ano pós-operatório de fixação in situ bilateral da cabeça femoral por ECFP. Ela relatava dor e limitação de movimentos do quadril esquerdo que se agravava com o apoio.

Durante a inspeção, foi observada atitude em rotação externa do membro inferior esquerdo, mais evidente durante a deambulação. A paciente apresentou leve claudicação em membro inferior esquerdo durante a marcha, a qual foi associada ao quadro de dor no quadril. Não foi observado sinal de Trendelenburg.

Ao exame físico, a paciente apresentava limitação importante da rotação interna do quadril esquerdo associada a quadro álgico durante a manobra. Foi evidenciado sinal de Drennan à esquerda durante o exame. A paciente não apresentava alterações neurovasculares nos membros inferiores, e tinha a força muscular preservada neles.

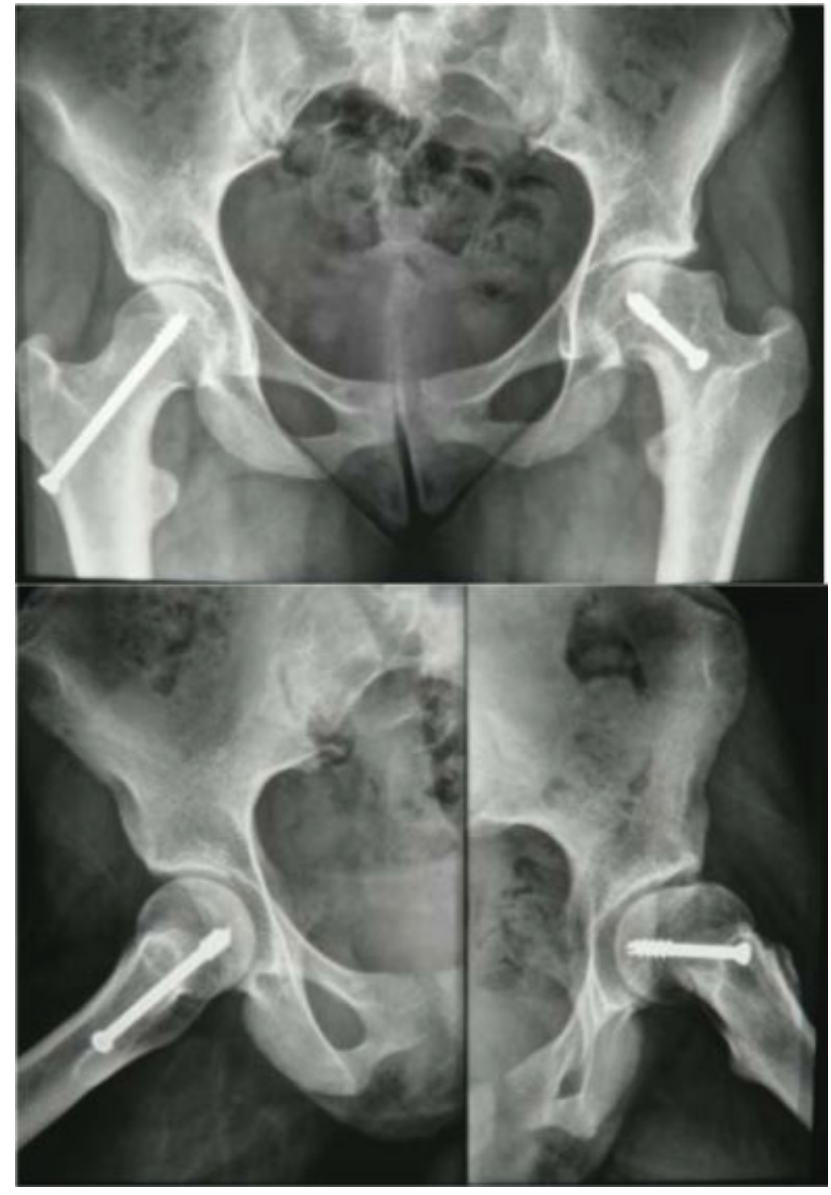

Fig. 1 Radiografias em incidência anteroposterior da bacia (acima) e de perfil de Dunn dos quadris (abaixo) evidenciando deformidade em região anterolateral do colo femoral esquerdo, compatível com impacto tipo came.

Nas radiografias em incidência anteroposterior (AP) da pelve e de perfil dos quadris (-Figura 1), foi observada epifisiólise do quadril esquerdo, com significativa proeminência anterolateral na transição cabeça-colo associada a

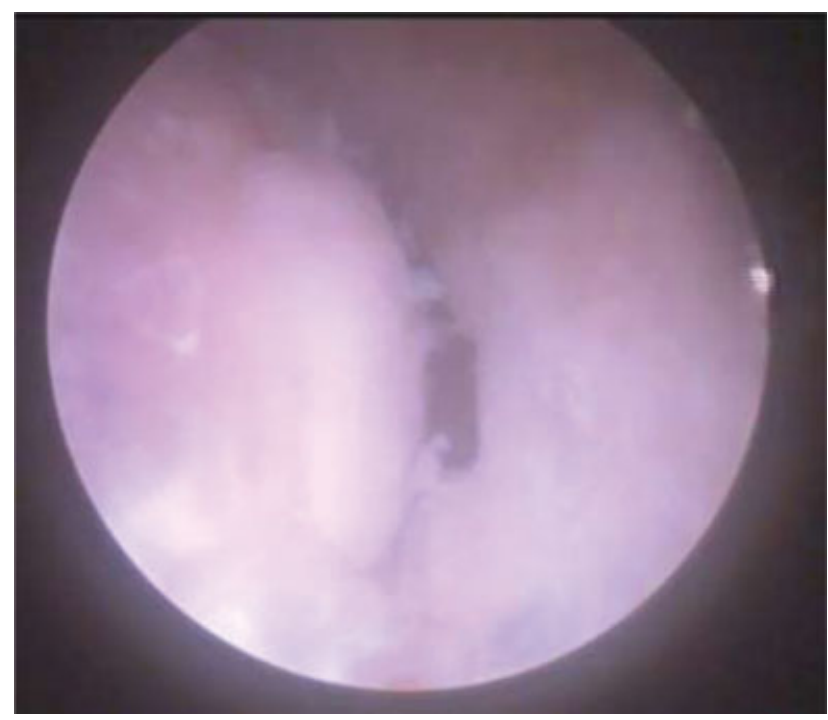

Fig. 2 Lesão condrolabral observada durante a artroscopia. 


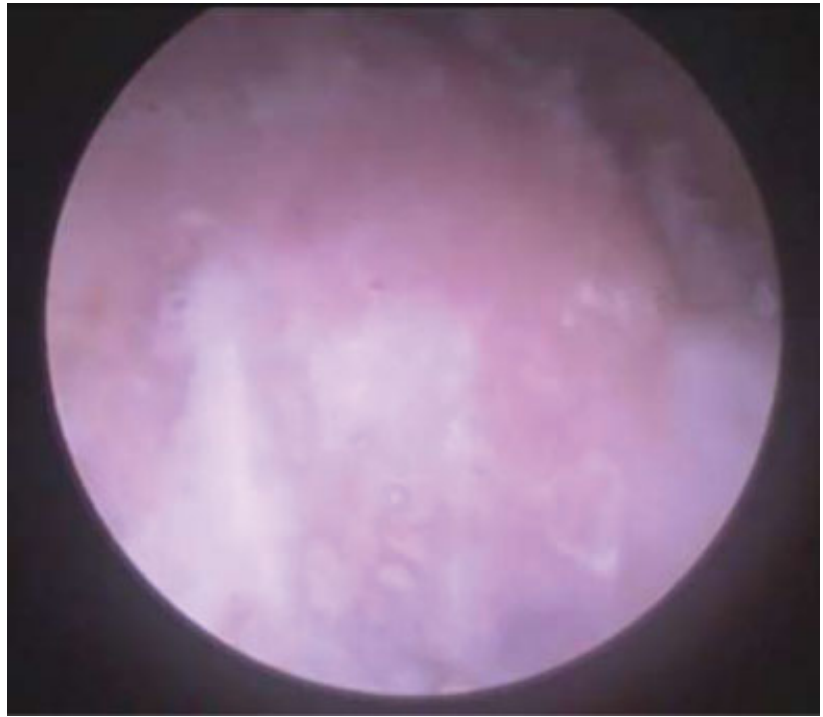

Fig. 3 Deformidade tipo came na transição cabeça-colo.

redução da compensação. O sinal de Trethowan estava presente. A fise de crescimento já se encontrava fechada.

A anamnese, o exame físico e as radiografias foram compatíveis com IFA tipo came, secundário ao quadro de epifisiólise. Devido ao quadro álgico sintomático associado a bloqueio articular, o tratamento preconizado foi a osteocondroplastia por via artroscópica.

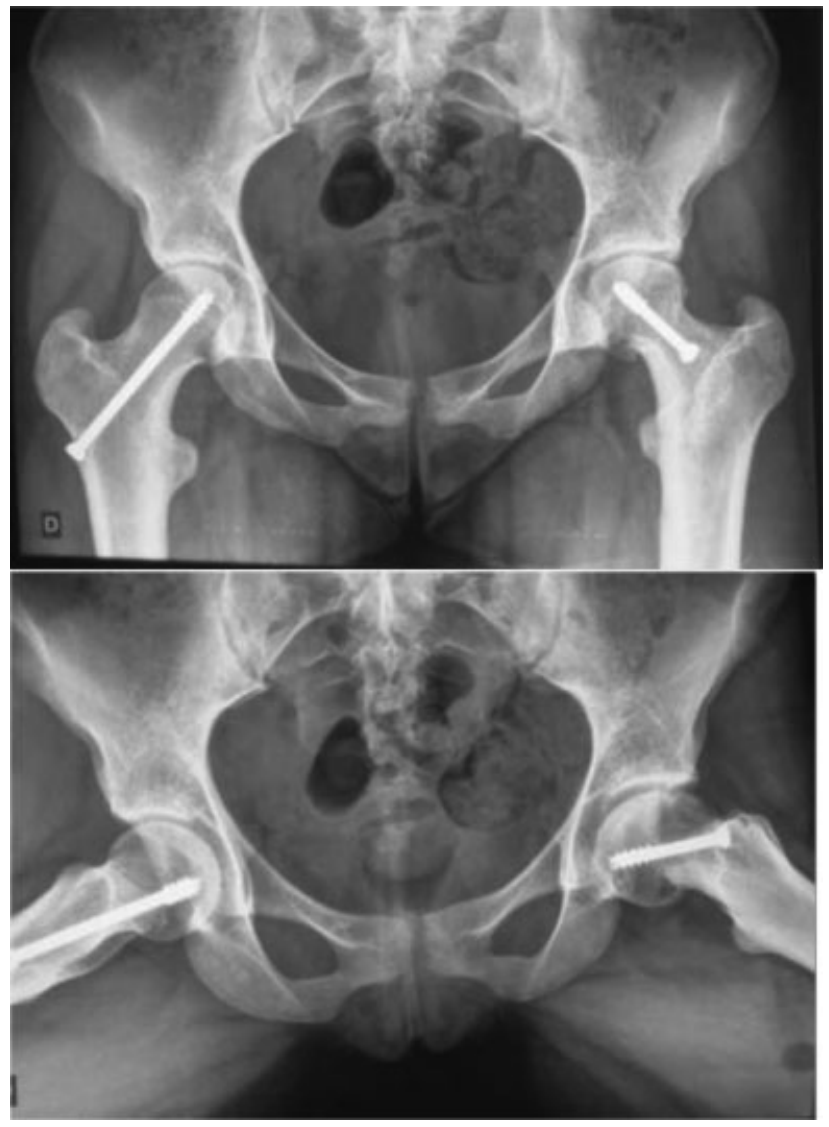

Fig. 4 Radiografia em incidência anteroposterior (acima) e de perfil de Lauenstein (abaixo) de bacia no pós-operatório evidenciando a correção da deformidade.
Durante a artroscopia, foi evidenciada lesão condrolabral no rebordo anterolateral do acetábulo (-Figura 2 ), compatível com IFA tipo came (-Figura 3), o qual foi confirmado durante a avaliação dinâmica no intraoperatório. Foi realizado desbridamento do lábrum e osteocondroplastia do colo femoral e do rebordo acetabular, com auxílio de fluoroscopia para controle da compensação cabeça-colo. Após o procedimento, a avaliação dinâmica não evidenciava mais impacto. As radiografias no pós-operatório demonstraram correção da proeminência responsável pelo impacto (-Figura 4)

Foi iniciada reabilitação no primeiro dia de pós-operatório, com movimentação passiva assistida e movimentação ativa, e deambulação com restrição de carga sobre o membro operado por duas semanas.

No primeiro mês de pós-operatório, a paciente já apresentava melhora significativa do quadro álgico e da marcha. Houve ganho importante da rotação interna do quadril esquerdo e da amplitude global de movimentos. Ao terceiro mês, ela deambulava sem queixas de dor. Ao sexto mês, retornou às atividades esportivas, encontrando-se totalmente assintomática.

\section{Discussão}

A associação entre ECFP, IFA sintomático e lesão condrolabral é atualmente bem estabelecida. ${ }^{8}$ Mesmo após a estabilização epifisária, casos específicos de ECFP podem ser adequados para o tratamento artroscópico, que consiste numa técnica emergente com poucos seguimentos em longo prazo. $^{9}$ Alguns estudos sugerem que a artroscopia pode ser aplicada mesmo em deformidades severas de epifisiólise. ${ }^{10}$

A queilectomia é um procedimento bem indicado para pacientes na faixa etária dos 10 aos 14 anos com sensação de bloqueio articular secundário a patologias do quadril na infância e adolescência, consistindo numa técnica relativamente simples e isenta de maiores complicações, podendo retardar o processo degenerativo da articulação por até 10 a 15 anos. $^{3}$

A seleção de pacientes que podem se beneficiar de uma artroscopia depende da morfologia femoral. A indicação precisa ainda não foi estabelecida, mas uma osteocondroplastia pode ser benéfica em casos de ECFPs associadas a impacto tipo came. Se as áreas de impacto da deformidade estiverem acessíveis a uma abordagem por via artroscópica, o cirurgião deve considerá-la em vez de uma abordagem aberta. Porém, o efeito mecânico dos diferentes graus de retroversão do colo femoral, a profundidade e a orientação acetabulares, e o deslocamento epifisário devem ser considerados antes de se indicar uma abordagem artroscópica. ${ }^{11}$

Conflito de Interesses

Os autores declaram não haver conflito de interesses.

\section{Referências}

1 Lehmann CL, Arons RR, Loder RT, Vitale MG. The epidemiology of slipped capital femoral epiphysis: an update. J Pediatr Orthop 2006;26(03):286-290 
2 Pritchett JW, Perdue KD. Mechanical factors in slipped capital femoral epiphysis. J Pediatr Orthop 1988;8(04):385-388

3 Guarnieiro R, Luzo CAM, Grigoletto Júnior W, et al. A queilectomia como operação de salvamento na patologia do quadril: resultados preliminares. Rev Bras Ortop 1995;30(1/2):42-44

4 Dodds MK, McCormack D, Mulhall KJ. Femoroacetabular impingement after slipped capital femoral epiphysis: does slip severity predict clinical symptoms? J Pediatr Orthop 2009;29(06): 535-539

5 Leunig M, Casillas MM, Hamlet M, et al. Slipped capital femoral epiphysis: early mechanical damage to the acetabular cartilage by a prominent femoral metaphysis. Acta Orthop Scand 2000;71 (04):370-375

6 Basheer SZ, Cooper AP, Maheshwari R, Balakumar B, Madan S. Arthroscopic treatment of femoroacetabular impingement following slipped capital femoral epiphysis. Bone Joint J 2016;98-B (01):21-27
7 Mahran MA, Baraka MM, Hefny HM. Slipped capital femoral epiphysis: a review of management in the hip impingement era. SICOT J 2017;3:35

8 Abraham E, Gonzalez MH, Pratap S, Amirouche F, Atluri P, Simon P. Clinical implications of anatomical wear characteristics in slipped capital femoral epiphysis and primary osteoarthritis. J Pediatr Orthop 2007;27(07):788-795

9 Leunig M, Horowitz K, Manner H, Ganz R. In situ pinning with arthroscopic osteoplasty for mild SCFE: A preliminary technical report. Clin Orthop Relat Res 2010;468(12):3160-3167

10 Akkari M, Santili C, Braga SR, Polesello GC. Trapezoidal bony correction of the femoral neck in the treatment of severe acuteon-chronic slipped capital femoral epiphysis. Arthroscopy 2010; 26(11):1489-1495

11 Zaltz I, Kelly BT, Larson CM, Leunig M, Bedi A. Surgical treatment of femoroacetabular impingement: what are the limits of hip arthroscopy? Arthroscopy 2014;30(01):99-110 